\title{
Effect on the Longitudinal Bending Deformation of Foam-Core Sandwich Superstructure When Material Parameters Alter
}

\author{
Bailu LUO ${ }^{1, \text { a }}$, Kai QIN ${ }^{2, \text { b }}$, Jie LV ${ }^{3, c}$ and Renjun YAN ${ }^{4, d}$ \\ ${ }^{1}$ China Ship Development and Design Center, Wuhan, China \\ ${ }^{2,4}$ Wuhan University of Technology, Wuhan, China \\ ${ }^{3}$ Wuchang Shipbuilding Industry Group CO., LTD, Wuhan, China \\ aluobailu2009@163.com, ${ }^{\text {b qinkai@whut.edu.cn, }{ }^{c} 1138859801 @ q q . c o m,{ }^{d} y a n \_r e n j u n @ 163 . c o m ~}$
}

\begin{abstract}
Keywords: Composite, Ship Superstructure, Material Parameters, Longitudinal Bending
Abstract. This paper analyzes how the deformation pattern of the superstructure's side wall alters when the material parameters of the laminate face sheet are tempered while the Foam-Core Sandwich Superstructure is under vertical and horizontal forces separately. The results revealed that, under each separate load of vertical forces and horizontal forces, the general pattern of longitudinal stress distribution is not affected by altering the elastic modulus of the face sheet; whereas its variation rate gradually increase as elastic modulus climbs. Comparatively, longitudinal stress distribution suffers from larger effect when the shear modulus is altered, and it presented different patterns with different loads: Under vertical forces, the variation rate of longitudinal stress grows, while it drops when under horizontal forces.
\end{abstract}

\section{Introduction}

Composite material has general superiority over conventional materials in its structural application in naval ships. It is light, magnetic-free, anti-erosion and enables structural functionality integration. The advantages mainly stretch in the following two aspects: Firstly, comparatively light material largely reduces structural weight, which means more payload that boosts combat efficiency. Secondly, when the severe operational environment of naval ships is considered, better anti-erosion character reduces maintenance time and cost, improves combat readiness rate, which actually cuts down full-cycle cost despite its initial high construction cost.

Through years of research and development, composite material has gained superiority especially on special purpose applications and taken an irreplaceable role in modern ship building industry. Composite is being widely used in constructing the superstructure of middle and large scale surface battleships, which reduces structural weight, betters global weight distribution, improves stability and increases surface physical protection. High performance crafts and major naval crafts are gradually adopting advanced composite materials in their manufacturing.

This paper combines finite element analysis along with decomposition method and analyzes how the deformation pattern of the superstructure's side wall alters when the material parameters of the laminate face sheet are tempered. The results can provide some reference for sandwich composite superstructure's structural design.

\section{Calculation model}

Discrete model analyses. The main hull and the superstructure together make up the whole ship hull girder, whose stress and deformation patterns should be analyzed under reasonable assumptions. Based on the decomposing thought, the following two methods are usually referred to when dealing with the strength analyses of ship superstructures. (1) The hull girder is divided into three parts: the main hull, the superstructure decks and the side walls of the superstructure. (2) The hull girder is divided into two parts: the main hull and the superstructure. In this paper, the second method is adopted, and combination beam theory is also applied, as is shown in Fig.1. 


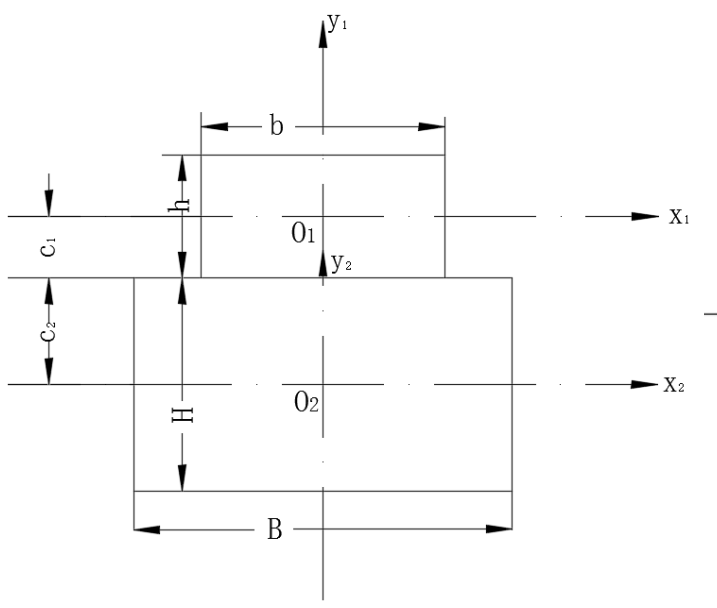

(a)

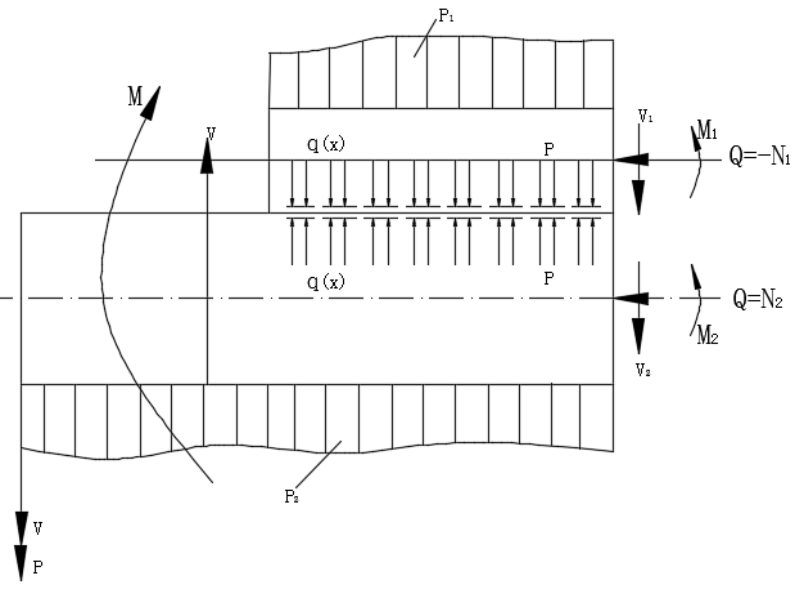

(b)

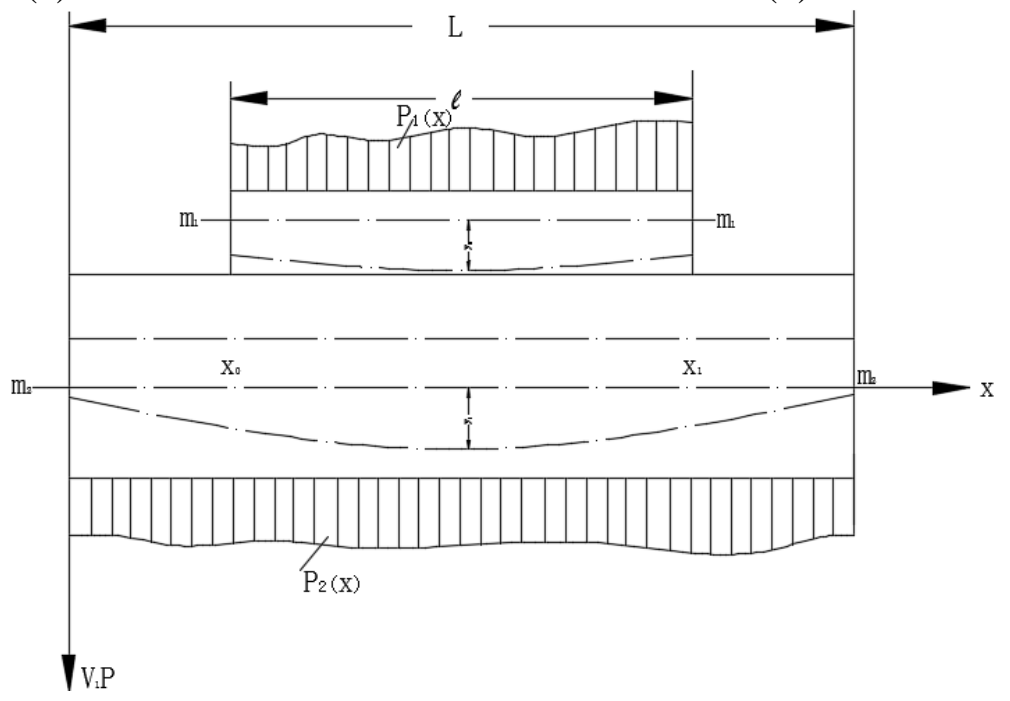

(c)

Fig.1 Calculation illustrations of combination beam consists of main hull and superstructure

On the contact surface that links main hull and superstructure, the forces can be decomposed into the vertical force $\mathrm{p}$, and horizontal force $\mathrm{q}$. The vertical force $\mathrm{p}$ wants the superstructure to bend along with the main hull. The horizontal force q tries to bend the superstructure opposite to the main hull, and passes on longitudinal forces at the same time. Base on this analyses, this paper independently calculated the superstructure, and following two border conditions are applied to the contact surface separately.

Vertical displacements are applied to the nodes of lower edge of the superstructure. The values of the displacement are the same with the whole model of the relevant notes, while the other two directions of displacements are zero. This simulates the effect of the vertical force $\mathrm{p}$ on the superstructure independently. The calculated longitudinal forces are represented with $\sigma_{x p \text {., }}$

Horizontal displacements are applied to the nodes of lower edge of the superstructure. The values of the displacement are the same with the whole model of the relevant notes, while the other two directions of displacements are zero. This simulates the effect of the horizontal force $\mathrm{q}$ on the superstructure independently. The calculated longitudinal forces are represented with $\sigma_{x q}$.

Finite element model. In the complete model, the calculation model includes the full superstructure and the main hull, which has been substituted with a hollow girder equivalently. In the superstructure longitudinal range, the main hull extends each to the nearest transverse bulkhead. ANSYS. Is used to construct the whole composite superstructure and the main hull that is based on steel material. Local structural parts like toggle plates, as well as holes in girders and plates are not counted into the model. 
The outer plates of the steel main hull, decks and bulkheads are simulated with SHELL63 element. The framework of the main hull is composed of T-sections, angle steels and flat steels, which are simulated with BEAM188 element.

The sandwich plates in the composite superstructure are simulated with 4-node 3D SHELL181 element. Each node of this element has 6 degrees of freedom, and has the ability to include large-strain full-nonlinear calculation. The stiffened ribbings of the composite superstructure are simulated with BEAM188 elements while the PVC core material filled inside are neglected. Rigid areas are set on the front and after cross-section of the calculation model.

The coupling node are set with the same height with the neutral axis and corresponding fixed displacement constrains are applied.

\section{Effect on longitudinal stress distribution of the superstructure's side wall when the material parameters of the laminate face sheet are tampered}

This paper considers the effect on longitudinal stress distribution of the side wall of two superstructures with different lengths when the material parameters of the laminate face sheet are altered. The two lengths are 15\% (short superstructure) and $80 \%$ (long superstructure) of the whole ship length. The models are recorded as M-15\% and M-80\%. The material parameters of sandwich composite adopted in the full ship model are listed in Tab.1.

Tab.1 Layer parameters of the face sheet material

\begin{tabular}{|c|c|c|c|c|c|c|c|c|c|}
\hline Parameter & $\begin{array}{c}E_{1} \\
{[\mathrm{GPa}]}\end{array}$ & $\begin{array}{c}E_{2} \\
{[\mathrm{GPa}]}\end{array}$ & $\begin{array}{c}E_{3} \\
{[\mathrm{GPa}]}\end{array}$ & $v_{12}$ & $v_{13}$ & $v_{23}$ & $\begin{array}{c}G_{12} \\
{[\mathrm{GPa}]}\end{array}$ & $\begin{array}{c}G_{13} \\
{[\mathrm{GPa}]}\end{array}$ & $\begin{array}{c}G_{23} \\
{[\mathrm{GPa}]}\end{array}$ \\
\hline Value & 18.7 & 18.7 & 18.7 & 0.09 & 0.09 & 0.09 & 3.55 & 3.55 & 3.55 \\
\hline
\end{tabular}

Note: Subscript 1 and 2 refers to the fiber directions, Subscript 3 refers to the nomal direction.

Effect of elastic modulus (E). Single parameter method are applied in this part of research. Elastic modulus of the laminate face sheet is altered while its shear modulus and Poisson's ratio are unchanged. The elastic modulus taken are 27000MPa, 25000MPa, 23000MPa, 21000MPa, $19000 \mathrm{MPa}, 17000 \mathrm{MPa}, 15000 \mathrm{MPa}, 13000 \mathrm{MPa}, 11000 \mathrm{MPa}, 9000 \mathrm{MPa}, 7739 \mathrm{MPa}$. With each elastic modulus, the distribution curves of mid-section longitudinal stress of the superstructure's side wall in both M-15\% and M-80\% model are extracted and shown in Fig.2 \& Fig.3.

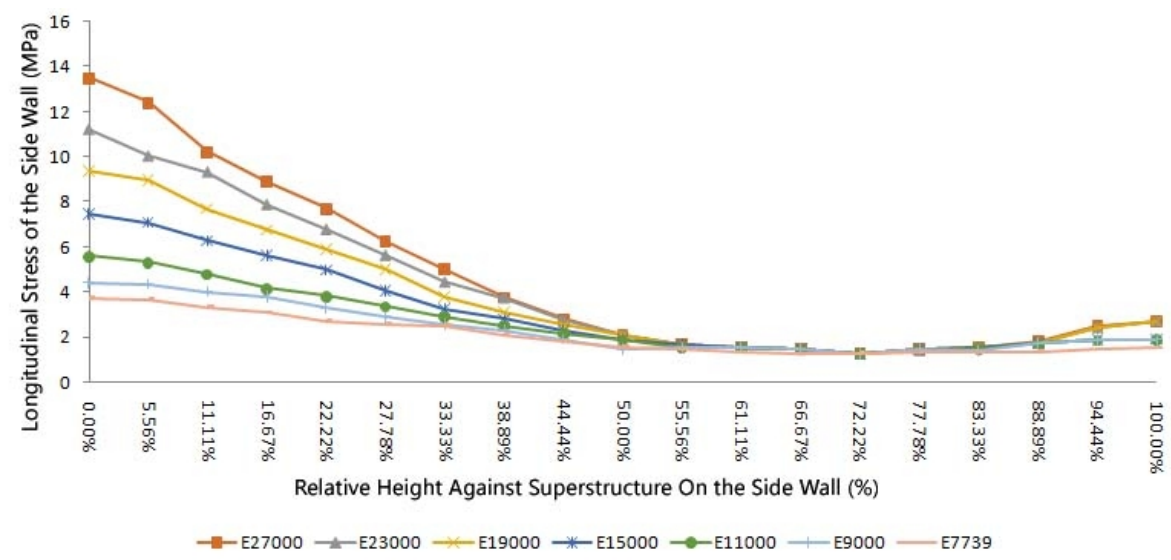

Fig.2 Side wall Longitudinal stress distribution along height of the M-15\% model with different E 


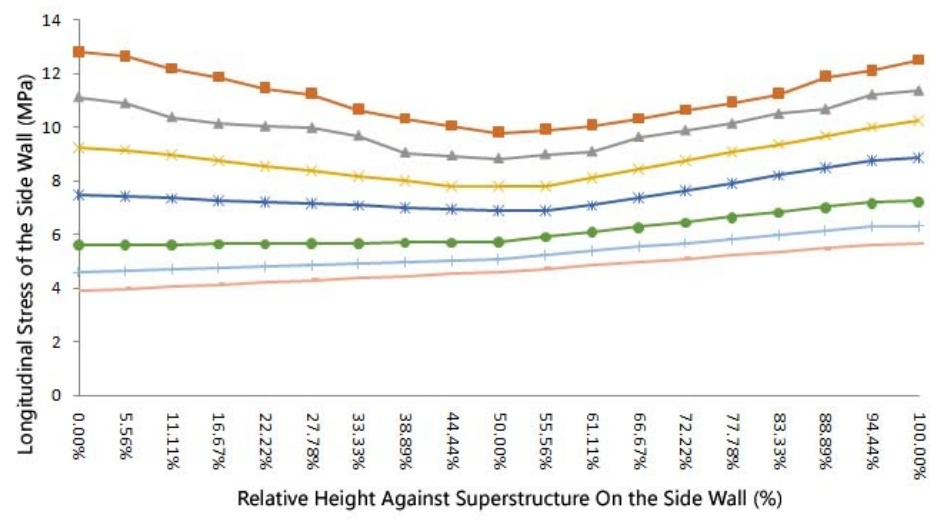

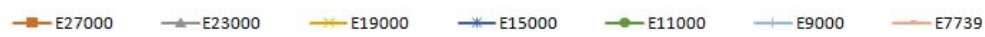

Fig.3 Side wall Longitudinal stress distribution along height of the M-80\% model with different $E$

It can be observed that, may it be the shorter superstructure and the longer one, the "turning point" of the stress curve all appears at $85 \%$ of the height of the superstructures' side wall. When the elastic modulus changes, the longitudinal stress distributions are greatly affected. As the elastic modulus gets smaller, the "turning point" of longitudinal stress curves gradually turns gentle. When the elastic modulus is $7739 \mathrm{MPa}$, which matches the equation $\mathrm{E}=2 \mathrm{G}(1+v)$ within material parameters, the curve nearly turns into a straight line. For the short superstructure, as the height increases past the stress "turning point", the change of elastic modulus no longer affects the longitudinal stress distribution in this region. But for the long superstructure, as the height increases past the stress "turning point", the deduction of elastic modulus tends to reduce the longitudinal stress on the side walls with the same amplitude.

Then the previously discussed decomposing method are adopted. The superstructure is separated from the whole model and two kinds of boundary conditions are applied to the superstructure simultaneously.

Vertical displacement constrains are applied to the lower edge of the independent superstructure. The values of displacement are the same with the whole ship model of the relevant notes. The other two directions of displacements are zero. The longitudinal stress calculated are expressed as $\sigma_{x p}$. Horizontal displacement constrains are applied to the lower edge of the independent superstructure. The values of displacement are the same with the whole ship model of the relevant notes. The other two directions of displacements are zero. The longitudinal stress calculated are expressed as $\sigma_{x p}$.

The vertical forces and horizontal forces are analyzed when the Elastic modulus of the laminate face sheet are tampered and its corresponding evolving patterns of longitudinal forces $\sigma_{x p}$ and $\sigma_{x q}$, shown in Fig.4 Fig.7.

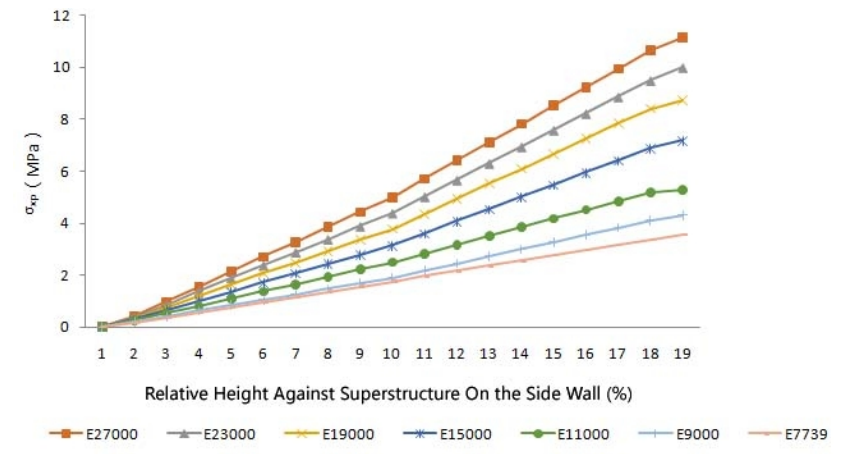

Fig. $4 \sigma_{x p}$ distribution along height of $M-15 \%$ model with different $E$

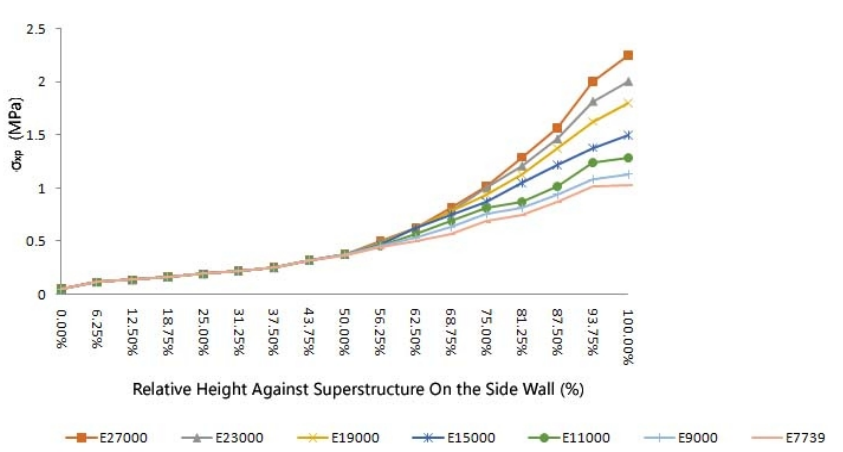

Fig. $5 \sigma_{x p}$ distribution along height of $M-80 \%$ model with different $E$ 


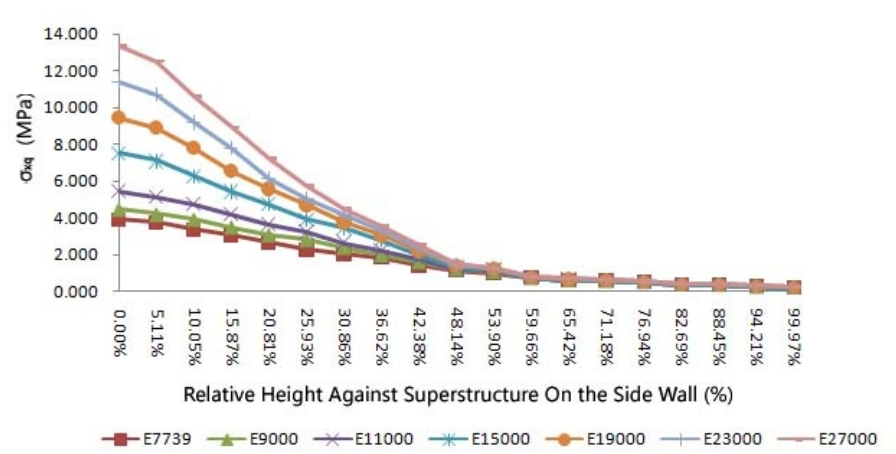

Fig. $6 \sigma_{x q}$ distribution along height of $M-15 \%$ model with different $E$

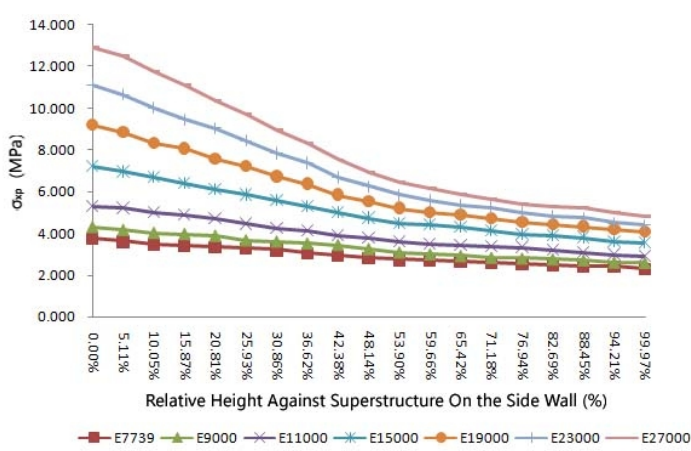

Fig. $7 \sigma_{x q}$ distribution along height of $M-80 \%$ model with different $E$

The curves in Fig.4 Fig.7. indicate that, when the superstructure is under the effect of vertical forces and horizontal forces separately, the alteration of elastic modulus of the face sheet renders the same effect on the longitudinal stress distribution on the side wall structure. As the elastic modulus increases, when the superstructure are merely under the effect of vertical forces, longitudinal stress on the side wall has a climbing trend. When the superstructure is merely ender the effect of horizontal forces, longitudinal stress on the side wall tends to decrease. However, the impact to short superstructure is not as significant as it renders on the long superstructure.

Effect of shear modulus (G). Shear modulus of the laminate face sheet is altered while its elastic modulus and Poisson's ratio are unchanged. The shear modulus taken are $3550 \mathrm{MPa}, 4000 \mathrm{MPa}$, $5000 \mathrm{MPa}, 6000 \mathrm{MPa}, 7000 \mathrm{MPa}, 8000 \mathrm{MPa}, 9000 \mathrm{MPa}, 10000 \mathrm{MPa}, 11000 \mathrm{MPa}, 12000 \mathrm{MPa}$, $12385 \mathrm{MPa}$. With each shear modulus, the distribution curves of mid-section longitudinal stress of the superstructure's side wall in both M-15\% and M-80\% model are extracted and shown in Fig.8 \& Fig.9.

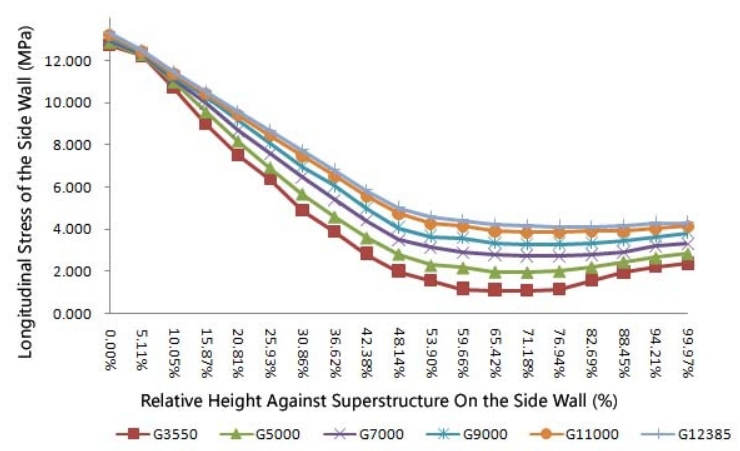

Fig8. Side wall longitudinal stress distribution of $M-15 \%$ model with different $G$

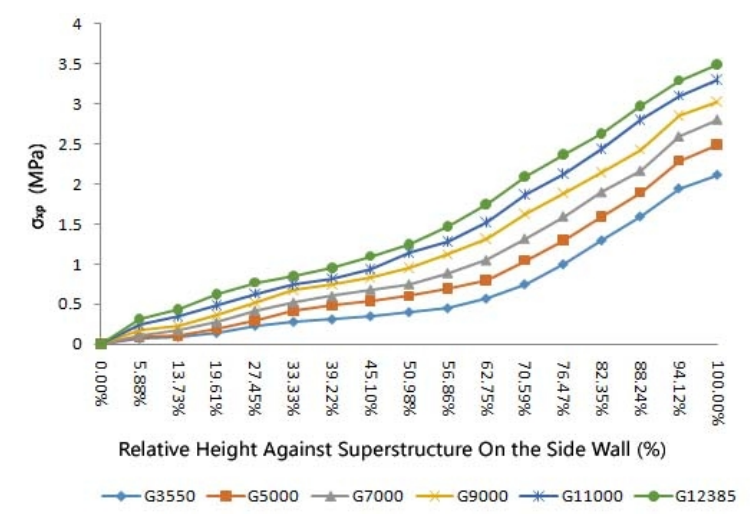

Fig. $10 \sigma_{x p}$ distribution along height of $M-15 \%$ model with different $G$

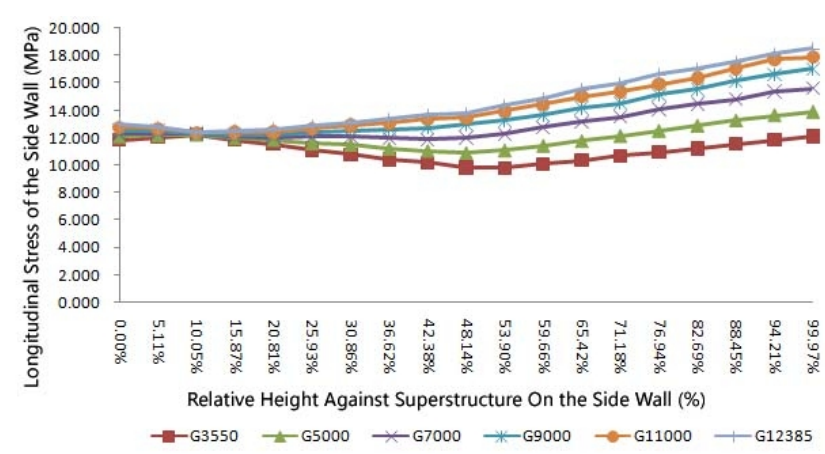

Fig.9 Side wall longitudinal stress distribution of $M-80 \%$ model with different $G$

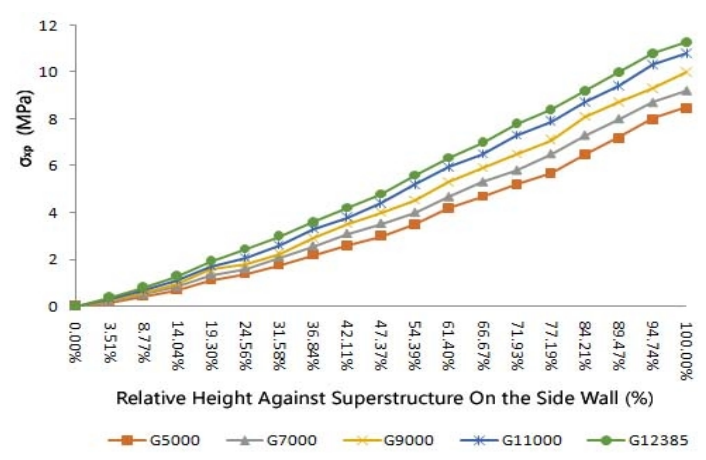

Fig.11 $\sigma_{x p}$ distribution along height of $M-80 \%$ model with different $G$ 


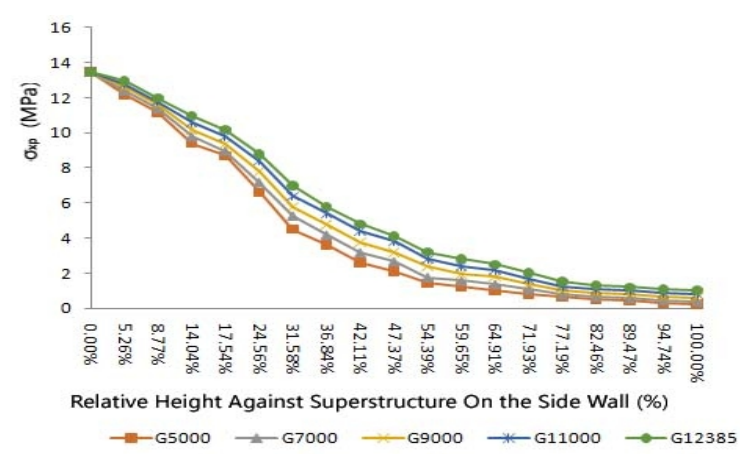

Fig.12 $\sigma x q$ distribution along height of $M-15 \%$ model with different $G$

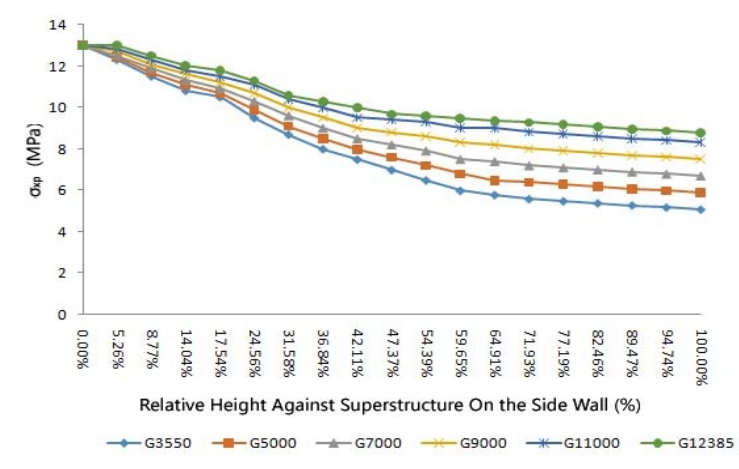

Fig. $13 \sigma_{x q}$ distribution along height of $M-80 \%$ model with different $G$

It can be drawn that the alteration of shear modulus presents the same effect on the longitudinal stress distribution patterns of the superstructure side wall. With the increase of shear modulus, the longitudinal stress level elevates until it reaches at a certain point that the elevating rate would decrease from its previous constant value. This means that for short superstructures, as the location on side wall keeps climbing, the longitudinal level will eventually fall into a constant level. But this is not the case for long superstructures. As the location on side wall climbs, the longitudinal stress level would stay unchanged for a little while, and then elevate almost linearly.

When the superstructures are separated independently, as the shear modulus of face sheet alters, the longitudinal stress curve of $\sigma_{x p}$ and $\sigma_{x q}$ corresponding to the vertical and horizontal forces are shown in Fig.10 13.

Effect of Poisson's ratio (v). While keeping the elastic modulus and shear modulus unchanged, Poisson's ratio is altered. Poisson's ratio is taken as $0.05,0.07,0.09,0.11,0.13,0.2,0.3$. With Poisson's ratio, the distribution curves of mid-section longitudinal stress of the superstructure's side wall are extracted and shown in Fig.14. \& Fig.15. The results show that the alteration of Poisson's ratio has no influence on the longitudinal stress distribution of both long and short superstructures.

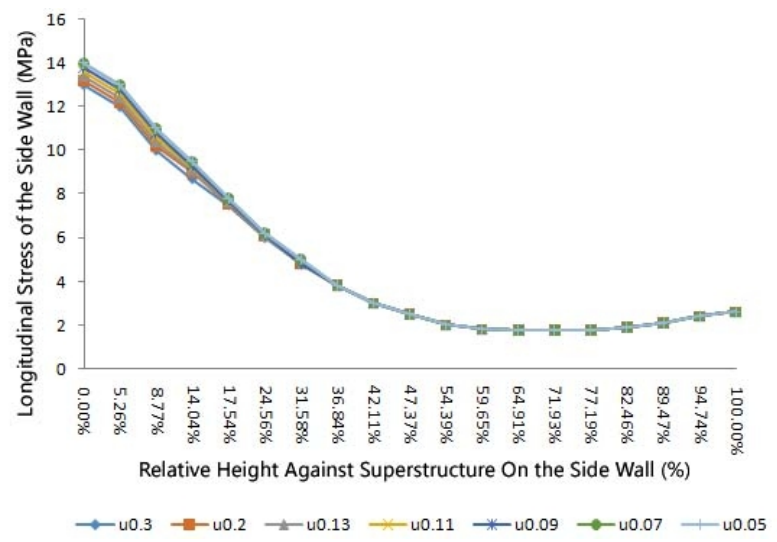

Fig.14 Side wall longitudinal stress distribution of $M-15 \%$ model with different $v$

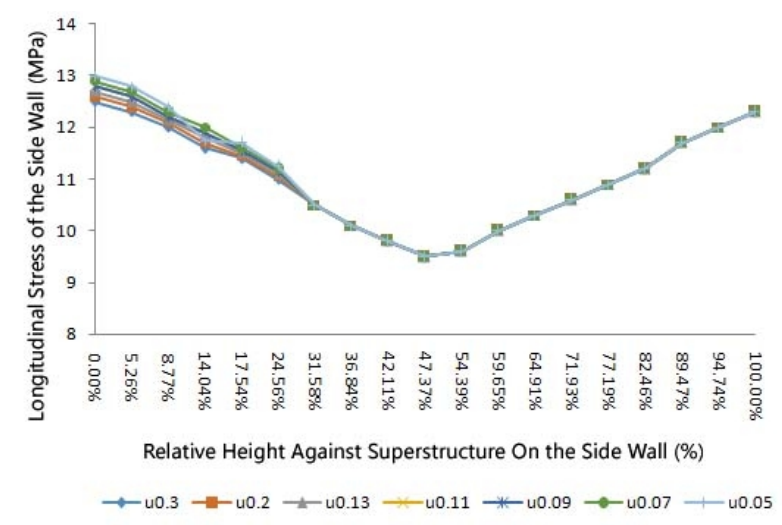

Fig.15 Side wall longitudinal stress distribution of $M-80 \%$ model with different $v$

\section{Conclusions}

This paper studies the foam core sandwich composite superstructures that is of $15 \%$ and $80 \%$ of the total ship hull length separately. The effect on longitudinal stress of the superstructure side walls is discussed when material parameters (Elastic modulus E, shear modulus G and Poisson's ratio $v$ ) of the sandwich face sheet are altered. Decomposing method is adopted to separate the superstructure from the main hull. The influence on longitudinal stress of the side wall of the superstructure is also analyzed 
when it is merely under the vertical forces or horizontal forces from the main hull separately. The following conclusions are drawn.

(1) Elastic modulus and shear modulus has relatively significant effect on the longitudinal stress distributions on the superstructure side wall, while Poisson's ratio renders little influence.

(2) The altering of elastic modulus poses less effect on the longitudinal stress distributions of the short superstructure than that of the long superstructure. But when the elastic modulus decreases, the "turning point" of stress distributions of superstructures with both lengths tend to ease, which means stress changing rate drops.

(3) The altering of shear modulus poses significant effect on the longitudinal stress distributions of both the short superstructure and the long one. When shear modulus increase, longitudinal stress of the side wall of both types of superstructure elevates at the same time. But under the same shear modulus, longitudinal stress of the short superstructure decreases as the height increase, while that of the long superstructure climbs.

(4) Elastic modulus and shear modulus share the same influence pattern towards the longitudinal stress of superstructure side walls and this pattern does not change with the length of the superstructure.

\section{References}

[1] Wang Xinye, Yang Fubiao and Zeng Jingcheng: Design Principle and Applications of Sandwich Structural Composite, Beijing: Chemical Industry Press (2007)

[2] Bian Fengquan: Research on Finite Element Analysis of Military Shelter Sandwich Plate, Xi 'an: Xidian University (2007)

[3] Hoff.NJ: Bending and Buckling of Rectangular Sandwich Plates, NACA TN 2225 (1950)

[4] Thomsen.O.T: Theoretical and Experimental Investigation of Local Bending Effects in Sandwich Plates, Composites Structure (1995) 30(1):85-101

[5] Tu Chinghua: General Equations of Sandwich Plates Under Transverse Loads and Edgewise Shears and Compressions, Acta Physica Sinica (1954) 10 (4): 395-412

[6] Cheng Guangmin, Ding Suozhu: Common Method and Development for Composite Honeycomb Sandwich Structure Calculation, Fiber Reinforced Plastics/Composites (1996) (2):11-13.

[7] Shell Group of Mechanic Laboratory, Institute of Mechanics, Chinese Academy of Sciences: Bending, Stability and Vibration of Sandwich Shell Plate, Beijing: Science Press (1977) 